\title{
Traceable Signatures using Lattices
}

\author{
Thakkalapally Preethi and Bharat Amberker \\ Department of Computer Science and Engineering, National Institute of Technology Warangal, India
}

\begin{abstract}
Traceable Signatures is an extension of group signatures that allow tracing of all signatures generated by a particular group member without violating the privacy of remaining members. It also allows members to claim the ownership of previously signed messages. Till date, all the existing traceable signatures are based on number-theoretic assumptions which are insecure in the presence of quantum computers. This work presents the first traceable signature scheme in lattices, which is secure even after the existence of quantum computers. Our scheme is proved to be secure in the random oracle model based on the hardness of Short Integer Solution and Learning with Errors.
\end{abstract}

Keywords: Traceable Signatures, Lattices, Short Integer Solution, Learning with Errors.

Received October 7, 2019; accepted May 5, 2020

https://doi.org/10.34028/iajit/17/6/15

\section{Introduction}

Group signatures, introduced by Chaum and Heyst [5], allow members to sign messages anonymously on behalf of their group. The identity of the signer is not revealed from signatures and can be verified by the group public-key. In-case of any dispute, a trusted party called group manager can trace the signature and reveal the identity of the signer. One of the applications of group signatures is cloud security [16].

If a particular group member is suspected of an illegal activity, then all the signatures generated by that member have to be detected. In group signatures, this is done by the group manager by opening all the signatures. This violates the privacy of all the group members and is inefficient (centralized opening by the group manager). To overcome these two drawbacks, Kiayias et al. [9] defined traceable signatures where in addition to the group manager opening the signatures individually, he can reveal the tracing trapdoor of a suspected group member to his agents and agents can detect all the signatures generated by that member without revoking the anonymity of remaining group members. This also improves scalability as agents can run in parallel compared to the traditional group signatures. Moreover in traceable signatures, a signer can provably claim the authorship of his own signatures. Since 2004, a few traceable signature schemes were proposed and these are insecure once quantum computers come into existence [17].

- Lattice-based Cryptography: Since the works of Regev [15] and Gentry et al. [7], lattice-based cryptography have been an exciting research area. It is a promising alternative to classical cryptography due to the following reasons: constructions based on lattices are secure even in the presence of quantum computers, involves simple operations and are based on worst-case hardness assumptions.
Gordon et al. [8] introduced the first lattice-based group signature scheme. Since then, several latticebased group signature schemes with different features were proposed [10,11, 12, 13, 14]. All these schemes are proved to be secure in random oracle model. Even in the random oracle model, the design of group signature schemes with different traceable mechanisms is a non-trivial problem. In particular, no lattice-based traceable signature scheme has been proposed so far.

- Contribution: We propose the first traceable signature scheme using lattices. Compared to the existing lattice-based group signature schemes, our scheme has following advantages:

- User tracing: Agents on receiving the trapdoor of a particular group member from group manager can open all the signatures generated by that member preserving the anonymity of other members.

- Group member can claim the ownership of its own previously generated signature preserving the privacy of remaining signatures generated by that member.

- Group members can join dynamically. Compared to existing lattice-based group signature schemes which support dynamic joining $[12,14]$ the size of group public-key is efficient by $\log \mathrm{N}$ factor, where $\mathrm{N}$ is the number of members in the group.

Our scheme satisfies the security requirements, defined by Kiayias et al. [9], based on the hardness of two average-case lattice problems: Short Integer Solution (SIS) and Learning with Errors (LWE).

- Construction Overview: To achieve dynamic joining, we adapt the joining protocol in [12] which allows the new members to sample their secret-keys and are validated by the group manager. If secret- 
keys are valid, the group manager issues the membership certificates. In our scheme, joining protocol is same as in [12] except during the membership certificate generation. In [12], to generate certificate, it uses the encoding function defined in [3] which consists of $\mathrm{O}(\log \mathrm{N})$ matrices in group public-key (gpk). To decrease the size of gpk, our scheme uses the encoding function defined in [1], which consists of 3 matrices. Group manager maintains a database that contains all the information about registered members.

During signature generation, signer $i$ generates the syndrome on its secret-key and its certificate. These syndromes are individually encrypted using Regev encryption scheme [7]. Commitment on syndrome formed by secret-key is generated using SIS function (one-way).

An interactive zero-knowledge protocol is constructed to prove signer is a valid group member, ciphertexts are well-formed and commitment generated on secret-key syndrome is the correct commitment. This protocol is repeated many times to make soundness error negligible and is made non-interactive using Fiat-Shamir heuristic [6]. Group manager possesses the secret-key of regev encryption scheme. To achieve signature opening, group manager decrypts the syndrome on secret-key and reveals the identity using the database (containing the syndromes of all secret-keys along with the identities). If all the signatures generated by a particular suspected user has to be revealed, then group manager generates the trapdoor of user $i$, syndrome on user $i$ certificate and an intermediate key that decrypts the ciphertext on this syndrome, and is given to the agents. Agents upon receiving trapdoor for user $i$, decrypts the ciphertext given in the signature to obtain the syndrome on certificate $i$ and matches with the syndrome given in the trapdoor. Thus, user tracing is achieved in our scheme. Signer can claim the signature as his own by generating the Non-Interactive Zero-Knowledge (NIZK) protocol that the commitment in the signature is generated by using its own secret-key. Verifiers check the validity of the protocol to verify signature claiming.

- Organization: In section 2, model of traceable signatures and cryptographic primitives in lattices is presented. Section 3 presents the interactive zeroknowledge protocol used in our work. Construction of our scheme and its security proofs are discussed in sections 4 and 5, respectively. Finally, section 6 concludes our work.

\section{Preliminaries}

\subsection{Traceable Signatures}

This section presents the model of traceable signature [9]. It consists of following nine algorithms.

- Setup: On input security parameter $n \in N$, a trusted party executes this algorithm and outputs the group public-key (gpk) and a group manager secret-key (gmsk).

- Join: It is an interactive protocol between Group Manager (GM) and user $i\left(U_{i}\right)$. At the end of the protocol $U_{i}$ obtains the secret-key $\sec _{i}$ and a membership certificate cert $_{i}$. GM appends the $U_{i}$ transcript transcript $_{i}$ to the database called transcripts, which is a private database containing the transcripts of all users.

- Sign: On input message $m$, secret-key $\sec _{i}$ and membership certificate cert $_{i}$ this algorithm generates the traceable signature $\sum$ on $m$.

- Verify: This algorithm returns 0 or 1 when group public-key $g p k$, message $m$ and signature $\sum$ are given as input.

- Open: Given a valid traceable signature $\sum, \mathrm{GM}$ using his own secret-key gmsk and the database transcripts outputs an identity of the signer.

- Reveal: Given an index $i$ of a group member along with its join transcript transcript $i_{i}$ GM using his own secret-key outputs the tracing trapdoor trace $_{i}$ of user $i$.

- Trace: Given a group public-key $g p k$, a valid signature $\sum$, and tracing trapdoor trace $_{i}$ of user $i$ as input, this algorithm return 1 or 0 .

- Claim: On input $g p k$, a message signature pair $\left(m, \sum\right)$ given by user $i$, user $i$ secret-key $\sec _{i}$ and its membership certificate cert $_{i}$ this algorithm returns the claim $\tau$ for an authorship of $i$ for signature $\sum$.

- Claim-Verify: Given a gpk, message-signature pair $(m, \Sigma)$ and claim $\tau$, it returns 1 or 0 .

- Correctness: A traceable signature scheme is correct if the following four conditions are satisfied with high probability in $n$, where $n$ is the security parameter. Let Sign $_{U}$, Reveal $_{U}$ and Claim $_{U}$ be the oracles of Sign, Reveal and Claim algorithms of user $U$ respectively.

a) Sign Correctness: For all $m$, Verify ( $m, g p k$, $\left.\operatorname{Sign}_{U}\right)=1$.

b) Open Correctness: For any $m$, Open $\left(\operatorname{Sign}_{U}, g p k, m\right.$, gmsk, transcripts $)=\mathrm{U}$.

c) Trace Correctness: For any $m$, Trace $\left(g p k, \operatorname{Sign}_{U}\right.$, Reveal $\left._{\mathrm{U}}\right)=1$ and for any $i^{\prime} \neq U$ Trace $\left(g p k\right.$, Sign ${ }^{\prime}$, Reveal $\left._{\mathrm{U}}\right)=0$.

d) Claim-Verify Correctness: For all $(m, \Sigma) \leftarrow \operatorname{Sign}_{U}$ Claim-Verify $\left(m, \sum\right.$, Claim $_{U}$, gpk $)=1$ 
Security model of traceable signatures was formalized in [9]. A traceable signature scheme is secure if it is secure against misidentification, anonymity and framing attacks. In all these attacks, adversary is given access to the certain oracles which share the following variables:

- State: contains transcripts, secret-keys and certificates of all members joined in the group. Sigs: set of members whose signatures are revealed by $Q_{s i g}$ query. Revs: set of members whose trapdoor is revealed by the $Q_{\text {reveal }}$ query. $N$ is the number of members in the group. $U^{(p)}$ : set of honest members in the group. $U^{(a)}$ : set of adversary controlled members in the group and $U^{(b)}$ : set of members added by the adversary acting asgroup Manager (GM).

Oracles which are given access to the adversary are:

- $Q_{y}$ : returns gpk. $Q_{s}$ returns gmsk. $Q_{a-j o i n}$ : In the join protocol, oracle acts as a group manager and adversary acts as a user. $Q_{b \text {-join: }}$ In the join protocol, adversary acts as a group manager and oracle acts as a user. When protocol in $Q_{a-\text {-join }}$ and $Q_{b \text {-join }}$ terminates, it adds user $i$ to $U^{(a)}$ and $U^{(b)}$ respectively and sets state $=$ state $\mathrm{II}\left(i\right.$, cert $t_{i}$, transcript $\left._{i}, \perp\right)$, transcripts $=$ transcripwts $\|(i$, transcript $_{i}$ ).

- $Q_{p-j o i n}$ Introduces honest users in the group and sets state and transcripts as in $Q_{b \text {-join }}$ query.

- $Q_{\text {sig: }}$ On input message $m$ and index $i$, this oracle returns the signature $\sum$, if an entry is found in state and adds $(i, m, \Sigma)$ to sigs. If no entry is found or $i \in U^{(a)}$ then, it returns $\perp$ and $Q_{\text {reveal }}$ : returns the output of Reveal ( $i$, transcripts) and adds $i$ to Revs. Outputs $\perp$ if $i \in U^{(b)}$ or does not exist.

- Misidentification attack: In this attack, adversary can control a set of users in the group through $Q_{a \text {-join }}$ query. It is allowed to observe the system while generating signatures and adding users through $Q_{\text {sig }}$ and $Q_{b \text {-join }}$ queries.In-addition, adversary is allowed to access $Q_{\text {reveal }}$ which reveals the tracing trapdoor of users. Finally, adversary has to generate a valid signature that is not opened or traced to a user controlled by the adversary. It can be clearly explained in the following experiment.

Experiment $\operatorname{Exp}_{A}^{\text {mis }}(n):(g p k, g m s k) \leftarrow \operatorname{Setup}\left(1^{n}\right)$; $(m, \Sigma) \leftarrow A\left(Q_{p-\text { join }}, Q_{a-\text { join }}, Q_{\text {reveal }}, Q_{\text {sig }}\right) ; \quad$ If $\operatorname{Verify}(m, \Sigma, g p k)=0$ then return $0 ; \quad$ If $O \operatorname{pen}(m, \Sigma, g m s k)=\mathrm{j} \notin U^{(a))}$ or $\quad \Lambda_{i \in U^{(a)}} \operatorname{Trace}(\Sigma$, $\operatorname{Reveal}(\mathrm{i}))=0$ then return 1; return 0; A traceable signature is secure against misidentification attacks if $\operatorname{Pr}\left[\operatorname{Exp}_{A}^{m i s}(n)=1\right]$ is negligible in $n$.
- Anonymity attack: This attack operates in two phases: play and guess. In play phase, adversary has access to $Q_{a-\text { join }}, Q_{p-\text { join }}, Q_{\text {sig }}$ and $Q_{\text {reveal }}$ through which it controls set of users, observes the system during addition of members and signature generation and can obtain the tracing information of any user. At the end of play phase, adversary chooses two honest users which are not input to $Q_{\text {reveal }}$ query and obtains signature generated by one of them. In the guess stage, adversary has to guess the identity of the signer. This can be explained with the following experiment.

Experiment

$\operatorname{Setup}\left(1^{n}\right) ;\left(\operatorname{aux}, m, i_{0}, i_{1}\right) \leftarrow$

$A\left(Q_{p-\text { join }}, Q_{a-\text { join }}, Q_{\text {reveal }}, Q_{\text {sig }}\right)$; If $i_{0} \notin U^{(p)}$ or $i_{1} \notin$ $U^{(p)}$ or $i_{0} \in$ Revsori $i_{1} \in$ Revsthen return $0 ; \quad b \leftarrow$ $\{0,1\}, \Sigma \leftarrow \operatorname{Sign}\left(g p k, m, \sec _{i_{b}}, \operatorname{cert}_{i_{b}}\right)$;

$b^{\prime} \leftarrow \mathrm{A}\left(\right.$ guess, aux, $\left.\Sigma: Q_{p-\text { join }}, Q_{a-j o i n}, Q_{\text {reveal }}, Q_{\text {sig }}\right) \mathrm{I}$ $\mathrm{f} \mathrm{b}=b^{\prime}$, then return 1; return 0 ; A traceable signature is said to be secure against anonymity attacks if for any probabilistic polynomial-time algorithm $A$, $\operatorname{Pr}\left[\operatorname{Exp}_{A}^{\text {anon }}(n)=1\right]-\frac{1}{2} \mid$ is negligible in $n$.

- Framing attacks: In this attack, adversary is allowed to control group manager through $Q_{S}$ query. It can observe the system through $Q_{b \text {-join }}$ and $Q_{\text {sig }}$ queries. The goal of the adversary is either to generate a signature that opens or traces to honest user or to claim the ownership of the signature generated by another user. It can be described by the following experiment.

Experiment $\quad \operatorname{Exp}_{A}^{f r a}(n):(g p k, g m s k) \leftarrow$ $\operatorname{Setup}\left(1^{n}\right) ;(m, \Sigma, \tau) \leftarrow \mathrm{A}\left(Q_{y}, Q_{S}, Q_{b-j o i n}, Q_{s i g}\right) ; \quad$ If $\operatorname{Verify}(m, \Sigma, g p k)=0$ then return $0 ; \quad$ If $O$ pen $(m, \Sigma, g m s k) \in$ $U^{(b)}$ or $\forall_{i \in U^{(b)}} \operatorname{Trace}(\Sigma, \operatorname{Reveal}(\mathrm{i}))=1$ then return 1; If $\quad \forall_{i \in U^{(b)}}(i, \Sigma) \in$ sigs and Claim Verify $(m, \Sigma, \tau, g p k)=1$ then return 1 ; return 0; A traceable signature is secure against framing attacks if for any probabilistic polynomial-time adversary $A$, $\operatorname{Pr}\left[\operatorname{Exp}_{A}^{f r a}(n)=1\right]$ is negligible in $n$.

\subsection{Lattices}

For any $m$ linearly independent vectors $\mathrm{B}=\left(b_{1}, \ldots, b_{\mathrm{m}}\right)$, lattice $\mathrm{L}(B)$ is defined as

$$
L(B)=\left\{\sum_{i=1}^{m} x_{i} b_{i}: x_{i} \in Z\right\} .
$$

For any positive real number $s$, discrete guassian distribution over lattice $\Lambda$ is defined as $\mathrm{D}_{\Lambda, \mathrm{s}}(x)=$ $\rho_{s}(x) / \rho_{s}(\Lambda)$ for any $x \in \Lambda$.

For any $m, n \geq 1, q \geq 2$, matrix $A \in Z_{q}^{n \times m}$, lattice $\Lambda^{\perp}(A)$ is defined as

$$
\Lambda^{\perp}(A)=\left\{e \in Z^{m}: A e=0 \bmod q\right\}
$$


For any $u \in Z_{q}^{n}$, coset of the lattice $\Lambda_{u}^{\perp}(A)$ is defined as

$$
\Lambda_{\boldsymbol{u}}^{\perp}(A)=\left\{\boldsymbol{e} \in Z^{m}: A \boldsymbol{e}=\boldsymbol{u} \bmod q\right\}
$$

In our work, we consider two average case lattice problems are Short Integer Solution (SIS) and Learning With Errors (LWE).

$\mathrm{SIS}_{\mathrm{n}, \mathrm{m}, \mathrm{q}, \beta}^{\mathrm{p}}$ : Given a uniformly random matrix $\mathrm{A} \in$ $\mathrm{Z}_{\mathrm{q}}^{\mathrm{n} \times \mathrm{m}}$, find the vector $\mathrm{x} \in \Lambda^{\perp}(\mathrm{A})$ such that $\|\mathrm{x}\|_{\mathrm{p}} \leq \beta$.

$\operatorname{LWE}_{n, q, \psi}$ : Let $n, m \geq 1, q \geq 2$ and $\psi$ be the probability distribution over Z. For $\in Z_{q}^{n}$, the distribution $A_{s, \psi}$ over $Z_{q}^{n} \times Z_{q}$ is obtained by sampling a uniform vector $a \in Z_{q}^{n}, e \in \psi$ and outputting the pair $\left(a, a^{T} s+e\right)$. The goal of $\operatorname{LWE}_{n, q, \psi}$ is to distinguish $m$ samples chosen according to $A_{s, \psi}$ from the $m$ samples chosen according to uniform distribution over $Z_{q}^{n} \times Z_{q}$.

\section{Underlying Zero-Knowledge Argument System}

Let $D, L$ be positive integers. Libert et al. [12] proposed an interactive zero-knowledge protocol for the relation $R$

$$
R=\left\{(P, y ; x) \in Z_{q}^{D \times L} \times Z_{q}^{D} \times \text { Valid: } P x=y \bmod q\right\}
$$

Where Valid is the subset of $\{-1,0,1\}^{L}$ satisfying the following two conditions:

$$
\text { 1) } x \in \text { Valid } \Leftrightarrow T_{\pi}(x) \in \text { Valid }
$$

2) If $\boldsymbol{x} \in$ Valid and $\pi$ is uniform in $S$ then $T_{\pi(x)}$ is uniform in Valid

Where $T_{\pi}$ is the permutation of $L$ elements and set $S$ is the permutation of $m$ elements.

This section presents the Zero-knowledge Argument of knowledge (ZKAoK) for the scheme in section 4. In detail, it presents ZKAoK that satisfies the following conditions:

- Signer $i$ is a certified group member i.e, he possess a valid secret-key $z_{i}$ and membership certificate cert $_{i}=\left(i, d_{i}, s_{i}\right)$

- The syndrome $v_{i}$ obtained using secret-key $z_{i}$ is correctly encrypted to ciphertext $c_{v_{i}}=\left(c_{1}, c_{2}\right)$.

- The syndrome $w_{i}$ obtained using cert $_{i}$ and $z_{i}$ is correctly encrypted to ciphertext $c_{w_{i}}=\left(c_{3}, c_{4}\right)$..

- Commitment $b_{1}$ is the correct commitment of $v_{i}$.

All the above conditions can be defined as a relation $R^{\prime}$.

- Definition 1: The relation $R^{\prime}$ is defined as

$$
\begin{gathered}
R^{\prime}=\left\{A, A_{1}, A_{2}, u, F, B, B_{1}, D, D_{0}, G_{0}, G_{3}, D_{1}, c_{1}, c_{2}, c_{3}, c_{4},\right. \\
\left.b_{1} ; i, z_{i}, v_{i}, w_{i}, d_{i}, s_{i}, s_{0}^{\prime}, s_{1}^{\prime}, x_{1}, x_{2}, x_{3}, x_{4}\right\}
\end{gathered}
$$

Where

$$
\begin{aligned}
A, A_{1}, A_{2}, B, F, D, G_{3} & \in Z_{q}^{n \times m}, D_{0}, D_{1} \in Z_{q}^{2 n \times 2 m}, F \\
& \in Z_{q}^{4 n \times 4 m}, B_{1}, G_{0} \in Z_{q}^{n \times 2 m}, u \in Z_{q}^{n}, c_{1}, c_{3}, c_{4} \\
& \in Z_{q}^{m}, c_{2} \in Z_{q}^{2 m} ; i \in[N], z_{i}, d_{i}, s_{i} \\
& \in[-\beta, \beta]^{2 m}, v_{i} \in Z_{q}^{4 \cdot n}, w_{i} \in Z_{q}^{2 n}, s_{0}^{\prime}, s_{1}^{\prime} \\
& \in[-b, b]^{n}, x_{1}, x_{3}, x_{4} \in[-b, b]^{m}, x_{2} \\
& \in[-b, b]^{2 m}
\end{aligned}
$$

Satisfying

$$
\begin{gathered}
A d_{1 i}+A_{1} d_{2 i}+i A_{2} d_{2 i}=u+\operatorname{Dbin}\left(w_{i}\right) \\
w_{i}=D_{0} \operatorname{bin}\left(v_{i}\right)+D_{1} s_{i} \bmod q \text { and } v_{i}=F z_{i} \bmod q \\
c_{v_{i}}=\left(c_{1}, c_{2}\right)=\left(B^{T} s_{0}^{\prime}+x_{1}, G_{0}^{T} s_{0}^{\prime}+x_{2}+\operatorname{bin}\left(v_{i}\right) \frac{q}{2}\right) \\
c_{w_{i}}=\left(c_{3}, c_{4}\right)=\left(B^{T} s_{1}^{\prime}+x_{3}, G_{3}^{T} s_{1}^{\prime}+x_{4}+\operatorname{bin}\left(w_{i}\right) \frac{q}{2}\right) \\
b_{1}=B_{1} \operatorname{bin}\left(v_{i}\right) \bmod q
\end{gathered}
$$

Since, Libert et al. [12] proposed an interactive zeroknowledge protocol for relation $R$, an interactive zeroknowledge protocol for relation $R^{\prime}$ can be generated by transforming the relation $R$ to relation $R$ (defined in Equation (1)).

\subsection{Transformation of $R^{\prime}$ to $R$}

To transform the relation to $R$, we transform Equations (2), (3), and (4) to the form $P x=y \bmod q$, and define a set Valid such that it satisfies the conditions (1) and (2). We define the sets and matrices which are used in the transformation.

- $B_{3 m}$ is the set of all vectors in $\{-1,0,1\}^{3 m}$ having equal number of $-1,0,1 . B_{2 l}$ is the set of all vectors in $\{0,1\}^{2 l}$ having hamming weight $l$.

- For any $\alpha>0$, one can define the sequence $\left(\alpha_{1}, \alpha_{2}, \alpha_{3}, \ldots, \alpha_{p}\right)$ such that $\sum_{i=1}^{p} \alpha_{i}=$ $\alpha$ where $p=\log \beta+1$ [11]. A matrix $H_{m, \alpha}$ is defined as $\left[\alpha_{1}, \alpha_{2}, \alpha_{3}, \ldots, \alpha_{p}\right] \otimes I_{m} \in Z^{m \times m p}$ and a matrix $H_{m, \alpha}^{*}$ is obtained by adding $2 m$ columns to $H_{m, a}$.

- We define the matrix $R_{1}$ as $R_{1}=I_{4 n} \otimes$ $\left.\left[1|2| 4|\ldots| 2^{\log q}-1\right\}\right]$ and $R_{2}$ as $R_{2}=I_{2 n} \otimes$ $\left[1|2| 4|\ldots| 2^{\log q}-1\right]$.

The following lemma is used in the transformation.

- Lemma 4 [13]: Let $m, O$ be positive integers and $\delta_{O}=\log O+1$. On input a vector $v \in[-0,0]^{m}$, extension and decomposition technique outputs a vector $v^{*} \in B_{3 m \delta_{O}}$ such that $H_{m, o}^{*} v^{*}=v$

Conversion of all the equations in definition 1 to $P x=$ $y \bmod q$ proceeds as follows:

- Transformation of Equation (2) to the appropriate form: Let $i d \in\{0,1\}^{l}$ is the binary representation of $i$ and $i d_{j}$ represents the $j$-th bit of $i d$. Let $y_{1}=\operatorname{bin}\left(v_{i}\right)$ and $y_{2}=\operatorname{bin}\left(w_{i}\right)$ and Equation (2) can be written as

$$
\begin{aligned}
& A d_{1 i}+A_{1} d_{2 i}+\sum_{i=1}^{l}\left(2^{l-i} A_{2}\right) i d_{i} d_{2 i}-D y_{2}=u \\
& D_{0} y_{1}+D_{1} s_{i}-R_{2} y_{2} \bmod q=0, R_{1} y_{1}-F z_{i}=0 \bmod q
\end{aligned}
$$


Apply lemma (4) to the vectors $d_{1 i}$ and $d_{2 i}$ to generate the vectors $d_{1 i}^{*}$ and $d_{2 i}^{*}$ respectively. Extend $y_{2} \in$ $\{0,1\}^{m}$ and $i d \in\{0,1\}^{l}$ to $\widehat{y_{2}}$ andid $^{*}$ such that $\widehat{y_{2}} \in$ $B_{2 m}$ and $i d^{*} \in B_{2 l}$. Now, Equation (5) is reduced to

$$
A^{*} x_{11}=u \bmod q
$$

Where

$$
A^{*}=\left[A H_{m, \beta}^{*}\left|A_{1} H_{m, \beta}^{*}\right| 2^{l-1} A_{2} H_{m, \beta}^{*}|\ldots .| 2^{0} A_{2} H_{m, \beta}^{*}|-D| 0^{n \times m}\right]
$$

And

$$
x_{11}=\left[d_{1 i}^{*}\left\|d_{2 i}^{*}\right\| i d_{1}^{*} d_{2 i}^{*}\|\ldots\| i d_{2 l}^{*} d_{2 i}^{*} \| \hat{y}_{2}\right]
$$

Similarly, Equation (6) is reduced to

$$
C x_{12}=0 \bmod q
$$

Where

$$
C=\left(\begin{array}{cc}
C_{1} & 0 \\
0 & C_{2}
\end{array}\right) \text { and } x_{12}=\left(\begin{array}{l}
t_{1} \\
t_{2}
\end{array}\right)
$$

And $C_{1}=\left[D_{0}\left|0^{2 n \times 2 m}\right| D_{1} H_{m, \beta}^{*}\left|-R_{2}\right| 0^{n \times m}\right] C_{2}=$ $\left[R_{1}\left|0^{2 n \times 2 m}\right|-F H_{m, \beta}^{*}\right], t_{1}=\left[\widehat{y_{1}}|| s_{i}^{*} \| \widehat{y_{2}}\right]$ and $t_{2}=$ $\left[\widehat{y_{1}} \| z_{i}^{*}\right]$. The vectors $s_{i}^{*}$ and $z_{i}^{*}$ are obtained by applying lemma (4) to $s_{i}$ and $z_{i}$ respectively and $\widehat{y_{1}}$ is obtained by extending $y_{1}$ such that $\widehat{y_{1}} \in B_{4 m}$.

We combine Equations (7), (8) to obtain

$$
P_{1}^{*} x_{1}^{*}=z_{1} \bmod q
$$

Where

$$
P_{1}^{*}=\left(\begin{array}{cc}
A^{*} & 0 \\
0 & C
\end{array}\right) x_{1}^{*}=\left(\begin{array}{l}
x_{11} \\
x_{12}
\end{array}\right) z_{1}=\left(\begin{array}{l}
u \\
0
\end{array}\right)
$$

- Transformation of Equation (3) to the required form: Equation (3) can be written as

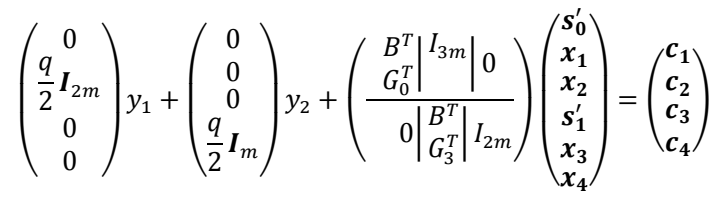

$$
\begin{aligned}
& \equiv Q_{1} y_{1}+Q_{2} y_{2}+Q_{3} t_{3}=z_{2}
\end{aligned}
$$

Apply lemma (4) to the vector $t_{3}$ to generate $t_{3}^{*} \in$ $B_{3(2 n+5 m) \delta_{b}}$ and $\widehat{y_{1}} \in B_{4 m}, \widehat{y_{2}} \in B_{2 m}$ is obtained by extending $y_{1}$ and $y_{2}$ respectively. Equation (10) can be written as

$$
P_{2}^{*} x_{2}^{*}=z_{2}
$$

Where

$$
\begin{gathered}
P_{2}^{*}=\left[Q_{1}\left|0^{5 m \times 2 m}\right| Q_{2}\left|0^{5 m \times m}\right| Q_{3} H_{m, b}^{*}\right], \\
\boldsymbol{x}_{2}^{*}=\left[\widehat{\boldsymbol{y}_{2}}|| \widehat{\boldsymbol{y}_{1}}|| \boldsymbol{t}_{\mathbf{3}}^{*}\right]
\end{gathered}
$$

- Transformation of Equation (4) to the required form: Let $B_{1}^{*}=\left[B_{1} \mid 0^{n \times 2 m}\right]$ and $\widehat{y_{1}} \in B_{4 m}$ is obtained by extending $y_{1} \in\{0,1\}^{2 m}$. Therefore, Equation (4) can be written as

$$
P_{3}^{*} x_{3}^{*}=z_{3}
$$

Where $P_{3}^{*}=B_{1}^{*}, x_{3}^{*}=\widehat{y_{1}}$ and $\mathrm{z}_{3}=\mathrm{b}_{1}$.

Finally, we combine the Equations (9), (11), and (12) as follows: Generate the matrix $P, x$ and $y$ as

$$
\mathrm{P}=\left(\begin{array}{ccc}
P_{1}^{*} & 0 & 0 \\
0 & P_{2}^{*} & 0 \\
0 & 0 & P_{3}^{*}
\end{array}\right) x=\left(\begin{array}{l}
x_{1}^{*} \\
x_{2}^{*} \\
x_{3}^{*}
\end{array}\right) \text { and } \mathrm{y}=\left(\begin{array}{l}
z_{1} \\
z_{2} \\
z_{3}
\end{array}\right)
$$

Thus, all the equations in relation $R^{\prime}$ (definition 1) are transformed to the form $P x=y \bmod q$.

Let $L=22 m+(2 l+4) 3 m \delta_{\beta}+3(2 n+5 m) \delta_{b}$.

We define set Valid as follows:

Valid: Set of all vectors $\{-1,0,1\}^{L}$ of the form

$g=\left[g_{1}|| g_{2}|| t_{1} g_{2}|| \ldots|| t_{2 l} g_{2}|| g_{3}|| g_{4}|| g_{5}|| g_{3}\left\|g_{4}\right\| g_{6}\right.$

$\left.g_{3}\left\|g_{4}|| g_{7}\right\| g_{4}\right]$

Where $g_{1}, g_{2}, g_{5}, g_{6} \in B_{3 m \delta_{\beta}}, g_{3} \in B_{2 m}, g_{4} \in$

$B_{4 m}, g_{7} \in B_{3(2 n+5 m) \delta_{b}}, t \in B_{2 l}$.

Let $\quad S=S_{3 m \delta_{\beta}} \times S_{3 m \delta_{\beta}} \times S_{2 l} \times S_{2 m} \times S_{4 m} \times$

$S_{3 m \delta_{\beta}} \times S_{3 m \delta_{\beta}} \times S_{3(2 n+5 m) \delta_{b}}$

Let $\pi=\left(\pi_{1}, \pi_{2}, \tau, \pi_{3}, \pi_{4}, \pi_{5}, \pi_{6}, \pi_{7}\right) \in S$.Define the permutation $T_{\pi}$ as

$T_{\pi}(g)=\left[\pi_{1}\left(g_{1}\right)\left\|\pi_{2}\left(g_{2}\right)\right\| t_{\tau(1)}\left(\pi_{2}\left(g_{2}\right)\right)\|\ldots\|\right.$.

$t_{\tau(2 l)}\left(\pi_{2}\left(g_{2}\right)\right)\left\|\pi_{3}\left(g_{3}\right)\right\| \pi_{4}\left(g_{4}\right)\left\|\pi_{5}\left(g_{5}\right)\right\| \pi_{3}\left(g_{3}\right) \|$

$\left.\pi_{4}\left(g_{4}\right)\left\|\pi_{6}\left(g_{6}\right)\right\| \pi_{3}\left(g_{3}\right)\left\|\pi_{4}\left(g_{4}\right)\right\| \pi_{7}\left(g_{7}\right) \| \pi_{4}\left(g_{4}\right)\right]$

By construction of vector $x$ in section 3.1, it belongs to set valid. It can be observed if a vector $x \in$ Validthen $T_{\pi(x)} \in$ Valid and vice-versa. Therefore, both the conditions (1 and 2) for valid set are satisfied. Since ZKAoK protocol for relation $R$ is given in [12] and our relation $R^{\prime}$ is transformed to $R$, ZKAoK protocol for relation $R^{\prime}$ is directly constructed from $R$.

\section{Proposed Scheme}

For any two matrices $A$ and $B$, concatenation of rows and columns are represented by $[A \mid B]$ and $[A \| B]$ respectively. Similar notation is also used for vectors.

We assume each user $U_{i}$ has public-key upk[i] and secret-key of $u p k[i]$ of a signature scheme as in [9]. Let $n$ be the security parameter, $N$ is the number of users joined the group, $m=2 n \log q, q=\tilde{O}\left(\ln ^{3}\right)$ and $q \gg$ $N, \sigma=\Omega(\sqrt{n \log q} \log n), \beta=\sigma \omega(\log m)$. Let $b=$ $\sqrt{n} \omega(\log n), t=\omega(\log n)$ and $\psi$ be the $b$-bounded distribution. We consider three random oracles $H:\{0,1\}^{*} \rightarrow\{0,1,2\}^{t}, \quad H_{1}:\{0,1\}^{*} \rightarrow Z_{q}^{n \times m} \quad$ and $H_{2}:\{0,1\}^{*} \rightarrow Z_{q}^{n \times 2 m}$. We use GenTrap and SamplePre algorithms presented in $[2,7]$ for our construction.

- $\operatorname{Setup}\left(1^{n}\right)$

1. Generate two instances of hard random lattices $\left(A, T_{A}\right)$ and $\left(B, T_{B}\right)$ using algorithm GenTrap $(n, m, q)$.

2. Choose matrices $\left(A_{1}, A_{2}, D\right)$ uniformly over $Z_{q}^{n \times m}$, $F$ is sampled uniformly from $Z_{q}^{4 n \times 4 m},\left(D_{0}, D_{l}\right)$ is uniformly chosen over $Z_{q}^{2 n \times 2 m}, B_{1}$ is uniformly chosen over $Z_{q}^{n \times 2 m}$ and vector $u$ is chosen uniformly over $Z_{q}^{n}$.

$g p k=\left(A, A_{1}, A_{2}, B, B_{1}, D, D_{0}, D_{1}, F, u\right)$ and $g m s k=$ $\left(T_{A}, T_{B}\right)$ 
Note: The size of gpk is $O(n m \log q)$.

Join $\left(G M, U_{i}\right)$.

1. User $U_{i}$ chooses a vector $z_{i} \leftarrow D_{Z^{4 m}, \sigma}$ and compute a vector $v_{i}=F z_{i} \bmod q$. Generate a signature on $v_{i}$ i.e., $\operatorname{sig}_{i}=\operatorname{sign}_{u s k[i]}\left(v_{i}\right)$. Send $v_{i}$ and $\operatorname{sig}{ }_{i}$ to GM.

2. GM verifies $s i g_{i}$ is valid signature of vector $v_{i}$ using $u p k[i]$ and was not previously generated by another user. If it is valid, then GM sets $i=N+1$ and computes user dependent matrix $A_{i}$ as $A_{i}=$ $\left[A \mid A_{1}+i A_{2}\right] \in Z_{q}^{n \times 2 m}$ and short $\quad$ vector $d_{i}=$ $\left[d_{1 i} \| d_{2 i}\right] \in Z^{2 m}$ such that

$$
A_{i} d_{i}=u+u_{i} \bmod q
$$

Where $u_{i}=D \operatorname{bin}\left(D_{0} \operatorname{bin}\left(v_{i}\right)+D_{1} s_{i}\right)$ and $s_{i}$ is chosen according to $D_{Z^{2 m}, \sigma}$ and send $\left(i, d_{i}, s_{i}\right)$ to $U_{i}$

3. $U_{i}$ checks whether $\left(i, d_{i}, s_{i}\right)$ satisfies Equation (14), $\left.|| d_{j i}\right|_{\infty} \leq \beta$ for $j \in\{1,2\}$ and ||$s_{i} \|_{\infty} \leq \beta$

If the conditions are valid then, $\sec _{i}=z_{i}, \operatorname{cert}_{i}=$ $\left(i, d_{i}, s_{i}\right)$ and stores the transcript $_{i}=$ $\left(\operatorname{sig}_{i}, v_{i}, i, d_{i}, s_{i}, u p k[i]\right)$ in the database transcripts which is the private database of GM.

Sign (gpk, $\left.\operatorname{cert}_{i}, \sec _{i}, m\right)$

1. Generate the one-time signature key-pair $(V K, S K)$ Compute $v_{i}=F z_{i} \bmod q$ and $w_{i}=D_{0} b i n\left(v_{i}\right)+D_{1} s_{i} \bmod q$.

2. Encrypt the vector $v_{i}$ using dual Regev Encryption scheme [7]. Let $G_{0}=\mathrm{H}_{2}(V K)$. Choose $s_{0}^{\prime} \leftarrow$ $\psi^{n}, x_{1} \leftarrow \psi^{m}$ and $x_{2} \leftarrow \psi^{2 m}$.

$$
c_{v_{i}}=\left(c_{1}, \mathrm{c}_{2}\right)=\left(B^{T} s_{0}^{\prime}+x_{1}, G_{0}^{T} s_{0}^{\prime}+x_{2}+\operatorname{bin}\left(v_{i}\right)\left(\frac{q}{2}\right)\right)
$$

3. Similarly, encrypt the vector $\mathrm{w}_{\mathrm{i}}$ Let $G_{1}=\mathrm{H}_{1}\left(\right.$ cert $\left._{i}\right)$. Choose $\mathrm{s}_{1}^{\prime} \leftarrow \psi^{\mathrm{n}}$ and compute $\mathrm{G}_{2} \in \mathrm{Z}_{\mathrm{q}}^{\mathrm{m} \times \mathrm{n}}$ such that $\mathrm{G}_{2} \mathrm{~s}_{1}^{\prime}=0 \bmod \mathrm{q}$ and proceed if one such $\mathrm{G}_{2}$ is found otherwise repeat. Let $G_{3}=G_{1}+G_{2}^{T}$. Choose $x_{3}, x_{4} \leftarrow$ $\psi^{\mathrm{m}}$

$$
c_{w_{i}}=\left(c_{3}, c_{4}\right)=\left(B^{T} s_{1}^{\prime}+x_{3}, G_{1}^{T} s_{1}^{\prime}+x_{4}+\operatorname{bin}\left(w_{i}\right)\left(\frac{q}{2}\right)\right)
$$

4. Generate the commitment for $\boldsymbol{v}_{i}$ as

$$
b_{1}=B_{1} \operatorname{bin}\left(v_{i}\right) \bmod q
$$

5. Generate a NIZK protocol $\Pi$ to prove there exists $i \in$ $[\mathrm{N}],\left(\mathrm{z}_{\mathrm{i}}, \mathrm{d}_{1 \mathrm{i}}, \mathrm{d}_{2 \mathrm{i}}, \mathrm{s}_{\mathrm{i}}\right)$ has infinity bound $\beta,\left(s_{0}^{\prime}, s_{1}^{\prime}, x_{1}, x_{2}, x_{3}, x_{4}\right)$ has infinity bound $b$ and there exists $v_{i}$ and $w_{i}$ that satisfies Equations (2), (3) and (4). This can be generated by running the interactive protocol in section $3 \mathrm{t}$ times and converting it into non-interactive using Fiat-Shamir heuristic [6] i.e., $\Pi=(\mathrm{CMT}, \mathrm{CH}, \mathrm{RSP})$ where

CH $\left(\mathrm{ch}_{1} \ldots ., \mathrm{ch}_{\mathrm{t}}\right) \mathrm{H}\left(\mathrm{CMT}, \mathrm{m},\left\{\mathrm{c}_{\mathrm{i}}\right\}_{\mathrm{i}=1}^{4}, \mathrm{VK}, \mathrm{G}_{3}, \mathrm{~b}_{1}\right) \in$ $\{0,1,2\}^{\mathrm{t}}$.

6. Compute the one-time signature sig $=$ OSign (SK, $\left.\left(\left\{c_{i}\right\}_{i=1}^{4}, b_{1}, \Pi\right)\right)$

$$
\Sigma=\left(c_{v_{i}}, c_{w_{i}}, \Pi, V K, \operatorname{sig}, G_{3}, b_{1}\right)
$$

$\operatorname{Verify}(m, g p k, \Sigma)$.

1. Check whether protocol $\Pi$ is valid.

2. Check whether sig is a valid signature on $\left(\left\{c_{i}\right\}_{i=1}^{4}, b_{1}, \Pi\right)$ using $V K$.

Return 1 iff all the conditions are valid.

Open ( $\sum$, gpk, $m$, gmsk, transcripts)

1. Compute $G_{0}=\mathrm{H}_{2}(V K)$ Decrypt $c_{v_{i}}$ using $T_{B}$ as follows: Using $T_{B}$, compute a small-norm matrix $E_{0} \in Z^{m \times 2 m}$ such that $B E_{0}=G_{0} \bmod q$. Obtain $\operatorname{bin}\left(v_{i}\right)$ by computing $\left(c_{2}-\frac{E_{0}{ }^{T} c_{1}}{\frac{q}{2}}\right)$

2. Compute $v_{i}=R_{1} \otimes \operatorname{bin}\left(v_{i}\right)$ and search in the database transcripts for transcript $t_{i}$ in which $v_{i}$ is the entry. If such transcript is found output the signer $i$ otherwise output $\perp$.

\section{Reveal (gmsk, $i$, transcripts)}

1. Parse $\operatorname{gmsk}=\left(T_{A}, T_{B}\right)$ and obtain transcript $_{i}=$ $\left(\operatorname{sig}_{i}, v_{i}, i, d_{i}, s_{i}, \operatorname{upk}[i]\right)$ from the database transcripts. Compute $\quad w_{i}=D_{0} \operatorname{bin}\left(v_{i}\right)+$ $D_{1} s_{i} \bmod q$

2. Let $G_{1}=\mathrm{H}_{1}\left(i, d_{i}, s_{i}\right)$. Compute the small norm matrix $E_{i} \in Z^{m \times m}$ using $T_{B}$ such that $B E_{i}=G_{1} \bmod q$.

trace $_{i}=\left(w_{i}, E_{i}\right)$.

Trace (gpk, $\sum$, trace $\left._{i}\right)$

1. Parse trace $_{i}=\left(w_{i}, E_{i}\right)$ and signature $\Sigma=\left(c_{v_{j}}, c_{w_{j}}, \Pi\right.$, $\left.V K, \operatorname{sig}, G_{3}, b_{1}\right)$.

2. Decrypt $c_{w_{j}}$ using $E_{i}$ and obtain $\operatorname{bin}\left(w_{j}\right)$.

3. Compute $w_{j}=R_{2} \otimes \operatorname{bin}\left(w_{j}\right)$ and return 1 iff $w_{j}$ is equal to the $w_{i}$ which is given as a part of trace $_{i}$.

Claim ( $m, \sum$, sec $_{i}$, ,ert $_{i}$, gpk $)$

1. Parse the signature $\Sigma=\left(c_{v_{i}}, c_{w_{i}}, \Pi, V K, \operatorname{sig}, G_{3}, b_{1}\right)$

2. Generate the NIZK proof of knowledge $\pi$ that there exists $z_{i}$ such that

$$
b_{1}=B_{1} \operatorname{bin}\left(v_{i}\right) \bmod q \text { where } v_{i}=F z_{i} \bmod q
$$

This is possible only if user has the secret-key $z_{i}$ and generated the signature $\Sigma$.

Output: $\tau=\pi$

Claim Verify $(m, \Sigma, \tau$, gpk)

Parse $\tau=\pi$ and signature $\Sigma\left(c_{v_{i}}, c_{w_{i}}, \Pi, V K, \operatorname{sig}, G_{3}, b_{1}\right)$. Check the validity of protocol $\pi$ and return 1 if it is valid.

- Correctness:

- Sign Correctness: By completeness of protocol $\prod$ and correctness of one-time signature scheme, Verify algorithm returns 1 with high probability. 
- Open Correctness: By correctness of Dual-Regev Encryption Scheme [7], open algorithm returns the identity $U$ with high probability.

- Trace Correctness: We know $\operatorname{Sign}_{U}$ and Reveal $_{U}$ oracles generate the signature and tracing trapdoor of user $U$ respectively. By Reveal algorithm in section 4 , the tracing trapdoor is $\left(w_{U}, E_{U}\right)$. By correctness of dual Regev encryption scheme, $E_{U}$ returnsw $w_{U}$. Therefore, Trace $\left(g p k\right.$, Sign $_{U}$, Reveal $\left._{U}\right)=1$ is satisfied with high probability. Next, we need to prove Trace $\left(\right.$ gpk, Sign $_{i}$, Reveal $\left._{U}\right)=0$ for any $i^{\prime} \neq U$. During trace algorithm in section (4), we decrypt $\left(c_{3}, c_{4}\right)$ using $E_{U}$ and obtain $\operatorname{bin}\left(w_{i}\right)$. But, $\left(c_{3}, c_{4}\right)$ is the encryption of $w_{i}$ and $E_{U}$ which is the trapdoor of user $U$ does not decrypt to $w_{i}$ ' correctly. Assume decryption algorithm returns $w_{j}$. Since, $w_{U}$ is statistically close to uniform, the probability that $w_{j^{\prime}}=w_{U}$ is negligible. Therefore, Trace $\left(g p k, \operatorname{Sign}_{i}\right.$, Reveal $\left._{U}\right)=1$ is negligible for any $i^{\prime} \neq U$.

- Claim-Verify Correctness: By completeness of protocol $\pi$ generated by claim $_{U}$, Claim-Verify algorithm returns 1 for all $(m, \Sigma) \leftarrow S i g n_{U}$.

\section{Security}

\subsection{Misidentification Attacks}

- Theorem 2: Our scheme is secure against misidentification attacks based on the hardness of SIS assumption.

- proof: Assume, there exists an adversary $A$ breaking the security of our scheme against misidentification attacks with non-negligible probability. We construct an algorithm $B$ that solves SIS instance $\bar{A}=\left[\bar{A}_{1} \mid \bar{A}_{2}\right] \in Z_{q}^{n \times 2 m_{\text {with }}}$ non-negligible probability. A coin is uniformly chosen over $\{1,2\}$ coin $=1$ and $i^{*} \stackrel{\$}{\leftarrow}[N]$.

- Setup:

- Assign the matrix $A=\bar{A}_{1}$. Run Gen Trap $(n, m, q)$ and obtain $\left(A_{2}, T_{A_{2}}\right)$ and $\left(B, T_{B}\right)$. Sample the matrices $R, R^{\prime}$ uniformly over $\{-1,1\}^{m \times m}$ and compute $A_{1}=A R-i^{*} A_{2}$.

- Sample the matrices $D_{0}, D_{1}$ uniformly over $Z_{q}^{2 n \times 2 m}, D=\bar{A}_{2} R^{\prime}$, matrix $B_{1}$ over $Z_{q}^{n \times 2 m}$ and matrix $F \in Z_{q}^{4 n \times 4 m}$.

- Vector $e$ is chosen according to $\mathrm{D}_{Z^{m}, \sigma}$ and compute $u=$ Ae $\bmod q$.

Send $g p k=\left(A, A_{1}, A_{2}, B, B_{1}, D, D_{0}, D_{1}, u, F\right)$ to $A$

- Queries:
- $Q_{P-\text { join }}$ : Increments $N$ and compute $A_{N}=\left[A \mid A_{1}+\right.$ $\left.N A_{2}\right]$. Using $T_{A_{2}}$ obtain $d_{N}=\left[d_{N, 1} \| d_{N, 2}\right]$. Let $s_{N}$ is chosen according to $\mathrm{D}_{Z^{2 m}, \sigma}$ and $z_{N}$ are chosen according to $\mathrm{D}_{Z^{4 m}, \sigma}$. Let $\operatorname{cert}_{N}=\left(N, d_{N}, s_{N}\right), \sec _{N}=z_{N}$ and add $N$ to the set $U^{(p)}$.

- $Q_{a^{-} \text {join }}$ : When $A$ triggers join protocol by sending $v_{i}, B$ chooses $N$ such that $N \neq i^{*}$. When $A$ provides $\operatorname{sig}_{i}$ such that it is a valid signature on $v_{i}$ under upk[i]. Using $T_{A_{2}}$ obtain the vector $d_{N}=$ $\left[d_{N, 1} \| d_{N, 2}\right]$ such that $A_{N} d_{N}=u+$ $D\left(\operatorname{bin}\left(D_{0} \operatorname{bin}\left(v_{N}\right)+D_{1} s_{N}\right)\right) \bmod q$ where $s_{N}$ is chosen according to $\mathrm{D}_{Z^{2 m}}, \sigma$. Send $\operatorname{cert}_{N}=\left(N, d_{N}\right.$, $\left.s_{N}\right)$ to $A$ and add $N$ to the set $U^{(a)}$.

- $Q_{\text {sig }}$ : If $i \notin U^{(p)}$ or $i=i^{*}$ then abort. Otherwise, generate the signature $\sum$ on message $m$ using $\sec _{i}$.

- $Q_{\text {reveal }}$ : On input index $i$, if $i \notin U^{(p)}$ or $i=i^{*}$ then abort. Otherwise, algorithm $B$ searches in the database transcripts for the entry $\left(., ., i, d_{i}, s_{i},.\right)$. Using transcripts $_{i}$ obtain trace $_{i}$ and add $i$ to Revs.

- Forgery: A outputs $\left(\mathrm{m}^{*}, \Sigma^{*}\right)$ such that Verify $\left(\right.$ gpk, $\left.\mathrm{m}^{*}, \Sigma^{*}\right)=1 . \quad$ IfOpen $\left(\left(\mathrm{m}^{*}, \Sigma^{*}\right.\right.$, gmsk $)=\mathrm{j} \in$ $\mathrm{U}^{(\mathrm{a})}$ orj $\neq \mathrm{i}^{*}$ abort. Otherwise, parse $\Pi^{*}=$ (CMT, CH, RSP) and $\Sigma^{*}=$ $\left(\mathrm{c}_{\mathrm{v}_{\mathrm{j}}}{ }^{*}, \mathrm{c}_{\mathrm{w}_{\mathrm{j}}}{ }^{*}, \Pi^{*}, \mathrm{VK}^{*}, \mathrm{G}_{3}{ }^{*}, \mathrm{~b}_{1}{ }^{*}, \operatorname{sig}^{*}\right)$. A must have queried the random oracle $\mathrm{H}$ on input $\left(\mathrm{CMT}, \mathrm{m}^{*}, \mathrm{VK}^{*},\left\{\mathrm{c}_{\mathrm{i}}^{*}\right\}_{\mathrm{i}=1}^{4}, \mathrm{G}_{3}^{*}, \mathrm{~b}_{1}^{*}\right) \quad$ with high probability. Otherwise,

$$
\operatorname{Pr}\left[\{c h\}_{\mathrm{i}=1}^{t}=\mathrm{H}\left(\mathrm{CMT}, m^{*}, V K^{*},\left\{c_{i}^{*}\right\}_{\mathrm{i}=1}^{4}, G_{3}^{*}, b_{1}^{*}\right)\right] \leq \frac{1}{3^{\mathrm{t}}}
$$

Therefore with $\varepsilon-3^{-t}$ probability, there exists an index $\kappa^{*} \leq \mathrm{Q}_{\mathrm{H}}$. At this stage, algorithm $B$ runs $A$ with same input and random tape as in original execution. Pick $\kappa^{*}$ as the target point and replay $A$ many times with the same random tape and input. Each time, first $\kappa^{*}-1$ queries are answered as $r_{1}, \ldots, r_{\kappa^{*}-1}$ and from $\kappa^{*}$ th query the answers are uniformly chosen from $\{1,2,3\}^{\mathrm{t}}$. The Improved Forking Lemma [4] implies that, with probability greater than $\frac{1}{2}, B$ can obtain a 3-fork involving tuple $\left(\mathrm{CMT}, m^{*}, V K^{*},\left\{c_{i}^{*}\right\}_{\mathrm{i}=1}^{4}, G_{3}^{*}, b_{1}^{*}\right)$ and open to the $\operatorname{bin}\left(v_{i}^{*}\right)$ which is uniquely determined by $\left(c_{1}^{*}, c_{2}^{*}\right)$. Let the answers of $B$ with respect to the 3 -fork branches be

$$
\begin{gathered}
r_{\kappa^{*}}^{(1)}=\left(c h_{1}^{(1)}, \ldots, c h_{t}^{(1)}\right) r_{\kappa^{*}}^{(2)}=\left(c h_{1}^{(2)}, \ldots ., c h_{t}^{(2)}\right) \\
r_{\kappa^{*}}^{(3)}=\left(c h_{1}^{(3)}, \ldots ., c h_{t}^{(3)}\right) \\
\operatorname{Pr}\left[\exists j \in[t]:\left\{c h_{j}^{(1)}, c h_{j}^{(2)}, c h_{j}^{(3)}\right\}=\{1,2,3\}\right]=\left(1-\left(\frac{7}{9}\right)^{t}\right)
\end{gathered}
$$

If such $j$ exists, parse the 3 -forgeries corresponding to 3-fork branches to obtain $\left(\mathrm{RSP}_{j}^{(1)}, \mathrm{RSP}_{j}^{(2)}, \mathrm{RSP}_{j}^{(3)}\right)$. Given three different challenges and three valid responses for same commitment $\mathrm{CMT}_{j}$, using witness extractionprocedure, the witness $\left(j, d_{j}=\right.$ 
$\left.\left[d_{1 j} \| d_{2 j}\right], z_{j}, s_{j}\right)$ can be extracted. Algorithm $B$ aborts if $j \neq i *$. We know $A_{j} d_{j}=u+\operatorname{Dbin}\left(w_{j}\right) \bmod q$ where $w_{j}=D_{0} \operatorname{bin}\left(v_{j}\right)+D_{1} s_{j} \bmod q$. Therefore,

$$
\begin{gathered}
{\left[\overline{A_{1}} \mid \overline{A_{1}} R\right]\left[d_{1 j}|| d_{2 j}\right]=u+\operatorname{Dbin}\left(w_{j}\right) \bmod q} \\
\left.\overline{A_{1}}\left(d_{1 j}+R d_{2 j}-e\right)-\bar{A}_{2} R^{\prime} \operatorname{bin}\left(w_{j}\right)\right)=0 \bmod q
\end{gathered}
$$

Let $\bar{x}=\left[d_{1 j}+R d_{2 j}-e \|-R^{\prime} \operatorname{bin}\left(w_{j}\right)\right]$. Since the vector $u$ statistically hides $e$ in $\Lambda_{u}^{\perp}\left(\bar{A}_{1}\right), \bar{x} \neq$ 0. Therefore, $\bar{x}$ is a solution to SIS instance i.e., $\bar{A} \bar{x}=$ $0 \bmod q$ and $\|\bar{x}\| \leq \sqrt{m}(\beta(m+2)+m)$.

Coin $=2$

- Setup:

- Assign the matrix $A=\bar{A}_{1}$ and $D=\bar{A}_{2}$. Run GenTrap $(n, m, q)$ obtain $\left(A_{2}, T_{A_{2}}\right)$ and $\left(B, T_{B}\right)$. Compute $A_{1}=A R-i^{*} A_{2}$ where $R$ uniformly over $\{-1,1\}^{m \times m}$

- Sample the matrix $D_{0}$ uniformly over $Z_{q}^{2 n \times 2 m}$ and matrices $F, B_{1}$ are uniformly chosen over $Z_{q}^{4 n \times 4 m} \times Z_{q}^{n \times 2 m}$ respectively. Let $\left(D_{1}, T_{D_{1}}\right)$ is obtained using GenTrap $(2 n, 2 m, q)$. Let $A_{i^{*}}=$ $\left[A \mid A_{1}+i^{*} A_{2}\right]$.

- Let $d_{1 i^{*}}$ and $d_{2 i^{*}}$ are chosen according to $\mathrm{D}_{Z^{m}, \sigma}$. Compute $u=A_{i^{*}} d_{i^{*-}} \operatorname{Dbin}\left(c^{\prime}\right)$ where $c^{\prime}$ is uniformly chosen over $Z_{q}^{n}$.

- Send $g p k=\left(A, A_{1}, A_{2}, B, B_{1}, D, D_{0}, D_{1}, u, F\right)$ to A.

- Queries: $Q_{P-\text { join, }} Q_{\text {sig }}$ and $Q_{\text {reveal }}$ : Answer similarly as in coin $=1$. For $Q_{a^{-} \text {join }}$ query proceed as follows If $i \neq i^{*}$ then, proceed as in case of coin $=1$. If $i=i^{*}$ : Recall $d_{i^{*}}$ and $c^{\prime}$. If $A$ provides valid signature on $v_{i^{*}}$ then using $T_{D_{1}}$ obtain $s_{i^{*}}$ such that $D_{1} s_{i^{*}}=c^{\prime}$ $D_{0} \operatorname{bin}\left(v_{i^{*}}\right)$. Send $\operatorname{cert}_{i^{*}}=\left(i^{*}, d_{i^{*}}, s_{i^{*}}\right)$ to $A$.

- Forgery: $A$ outputs $\left(m^{*}, \Sigma^{*}\right)$ and abort if Open $\left(\left(m^{*}, \Sigma^{*}, g m s k\right)=\mathrm{j} \notin U^{(a)} \quad\right.$ or $\quad j \neq$ $i^{*}$.Proceed if Verify $\left(g p k, m^{*}, \Sigma^{*}\right)=1$, $\operatorname{Open}\left(\left(m^{*}, \Sigma^{*}, g m s k\right)=\mathrm{j} \in U^{(a)}\right.$ and $\Lambda_{i \in U^{(a)}}$ Trace $\left(\Sigma^{*}, \operatorname{Reveal}(i)\right)=0$. Using forking lemma and knowledge extractor we obtain $\left(d^{*}, z^{*}, s_{i^{*}}\right)$. Since Trace $\quad\left(\Sigma^{*}, \operatorname{Reveal}\left(i^{*}\right)\right)=0, \quad w^{*}=D_{0} b i n\left(v^{*}\right)+$ $D_{1} s^{*} \neq D_{0} \operatorname{bin}\left(v_{i^{*}}\right)+D_{1} s_{i^{*}}=w_{i^{*}}$ and $\quad$ we know $\left[\overline{A_{1}} \mid \overline{A_{1}} R\right]\left[d_{1}^{*}|| d_{2}^{*}\right]-\operatorname{Dbin}\left(w^{*}\right)=$ $\left[\overline{A_{1}} \mid \overline{A_{1}} R\right]\left[d_{1 i^{*}}|| d_{2 i^{*}}\right]-\operatorname{Dbin}\left(w_{i^{*}}\right)=u$. Therefore, $\bar{x}=\left[d_{1}^{*}-d_{1 i^{*}}|| R\left(d_{2}^{*}-d_{2 i^{*}}\right)|| w^{*}-w_{i^{*}}\right]$ is $\quad$ a solution to SIS instance i.e., $\bar{A} \bar{x}=0 \bmod q, \bar{x} \neq$ 0 , and $\|\bar{x}\| \leq \sqrt{m}(2 \beta+2 m \beta+1)$.

\subsection{Anonymity Attacks}

- Theorem3: Our scheme is secure against anonymity attacks based on the zero-knowledge property of NIZK protocol Mand hardness of LWE.
- Proof: To prove our scheme is secure against anonymity attacks, we define two games $G_{0}^{(b)}$ and $G_{7}$. Game $G_{0}^{(b)}$ is the original anonymity game where challenge signature is generated by one of the users and game $G_{7}$ is the anonymity game where challenge signature is generated independent of both the users. We show that challenge signatures generated in both these games are computationally indistinguishable. This is because, if signatures generated in both these games are indistinguishable, then the advantage of adversary guessing the signer is negligible. To prove the signatures generated in these two games are indistinguishable, we define intermediate $G_{1}^{(b)}, G_{2}^{(b)}, G_{3}^{(b)}, G_{4}^{(b)}, G_{5}^{(b)}$ and $G_{6}^{(b)}$.

- Game $G_{0}^{(b)}$ : This is the original anonymity game. In precise, challenger runs setup algorithm to generate (gpk,gmsk) and gives gpk to adversary A. Challenger answers all the queries of the adversary. At some point, $A$ sends the challenge message $m^{*}$ and two identities $i_{0}$ and $i_{1}$. Challenger uniformly chooses one of the identity $b \in\{0,1\}$ and generates the challenge signature $\Sigma^{*}=$ $\left(c_{v_{i_{b}}}^{*}, c_{w_{i_{b}}}^{*}, b_{1}^{*}, \Pi^{*}, \operatorname{sig}^{*}, V K^{*}, G_{3}^{*}\right)$. Finally, $A$ outputs the bit $b^{\prime} \in\{0,1\}$.

- Game $G_{1}^{(b)}$ : In this experiment, we slightly change Game $G_{0}^{(b)}$ as follows: At the beginning of the game, the challenger generates the one-time signature key pair $\left(V K^{*}, S K^{*}\right)$ which will be used in the challenge phase. If $A$ requests the opening of a valid signature $\Sigma^{*}=\left(c_{v_{j}}, c_{w_{j}}, \Pi, V K, G_{3}, b_{1}, s i g\right)$ where $V K=V K^{*}$ the challenger returns a random bit and aborts.

- Game $G_{2}^{(b)}$ : In this game, we program the random oracle $\mathrm{H}_{2}$ in the following way: at the beginning of the game, choose a uniformly random matrix $G_{0} \in$ $Z_{q}^{n \times 2 m}$ and set $\mathrm{H}_{2}\left(V K^{*}\right)=G_{0}$. From the adversary's view, the distribution of $G_{0}$ is statistically close to the one in the real attack game, as in [7]. As for other queries, for each fresh $\mathrm{H}_{2}$ queries on $V K$, the challenger samples small-norm matrices $E_{0} \in$ $\mathrm{D}_{Z^{m}, \sigma}^{2 m}$ and programs the oracle such that $\mathrm{H}_{2}(V K)=B E_{0} \bmod q$. The chosen matrices $E_{0}$ are retained for later use.

- Game $G_{3}^{(b)}$ : In this game, we program the random oracle $\mathrm{H}_{1}$ in the following way: At the beginning of the game, a uniform matrix $G_{1}^{*} \in Z_{q}^{n \times m}$ is uniformly chosen and in the challenge phase, set $\mathrm{H}_{1}\left(\operatorname{cert}_{i_{b}}\right)=G_{1}^{*}$. For other $\mathrm{H}_{1}$ queries, fresh query on inputcert $t_{i}$, challenger samples $E_{i}$ according to $\mathrm{D}_{Z^{m}, \sigma}^{m}$ and set $\mathrm{H}_{1}\left(\right.$ cert $\left._{i}\right)=B E_{i}$ mod $q$. Retain $E_{i}$ for later use. From the adversary view, the distribution of $G_{1}^{*}$. is same as in Game $G_{0}^{(b)}$. 
- Game $G_{4}^{(b)}$ : In this game, we modify the way of handling the open and reveal queries. Challenger uniformly chooses a matrix $B^{*}$ uniformly over $Z_{q}^{n \times m}$ and to answer any reveal query of user $i$, it recalls $E_{i}$, computes $w_{i}$ using cert $_{i}$ and returns as trace $_{i}$. To answer any open query recall $E_{0}$ generated in Game $G_{2}^{(b)}$.

- Game $G_{5}^{(b)}$ : In this game, we change the generation of challenge signature. Instead of generating NIZK protocol $\Pi^{*}$ using the witness, simulate the protocol and obtain $\Pi^{\prime}$. The challenge signature is $\Sigma^{*}=$ $\left(c_{v_{i_{b}}}^{*}, c_{w_{i_{b}}}^{*}, b_{1}^{*}, \Pi^{\prime}, s i g^{*}, V K^{*}, G_{3}^{*}\right) . \quad$ By $\quad$ zeroknowledge protocol of $\Pi^{*}$, the challenge signature generated in this game is computationally indistinguishable from signature generated in Game $G_{2}^{(b)}$

- Game $G_{6}^{(b)}$ : We change the way of generating the challenge signature. We modify the generation of challenge cipher texts $\left(c_{1}^{*}, c_{2}^{*}, c_{3}^{*}, c_{4}^{*}\right)$. Instead of using encryption scheme [7], return random ciphertexts

$$
\begin{aligned}
& \left(c_{1}^{*}, c_{2}^{*}\right)=\left(r_{1}, r_{2}+\operatorname{bin}\left(v_{i_{b}}\right) \frac{q}{2}\right) \\
& \left(c_{3}^{*}, c_{4}^{*}\right)=\left(r_{3}, r_{4}+\operatorname{bin}\left(w_{i_{b}}\right) \frac{q}{2}\right)
\end{aligned}
$$

Where the vectors $\left(r_{1}, r_{2}, r_{3}, r_{4}\right)$, are uniformly chosen over $\left(Z_{q}^{m} \times Z_{q}^{2 m} \times Z_{q}^{n} \times Z_{q}^{m}\right)$. The challenge signature generated in this game is computationally indistinguishable from $\sum^{*}$ in $\operatorname{Game} G_{5}^{(b)}$ based on the hardness of decision version of LWE assumption.

- Game $G_{7}$ : Finally, we make a slight modification in generation of $\Sigma^{*}$ compared to the previous game. Ciphertexts $\left(c_{1}^{*}, c_{2}^{*}, c_{3}^{*}, c_{4}^{*}\right)$ is uniformly sampled over $\left(Z_{q}^{m} \times Z_{q}^{2 m} \times Z_{q}^{n} \times Z_{q}^{m}\right)$. Signature generated in this game is indistinguishable from the signature in previous game.

Challenge signature $\Sigma^{*}$ in the last game is independent of bit $b \in\{0,1\}$. Therefore, advantage of adversary in this game is 0 . We proved that the challenge signature generated in game $G_{7}$ is computationally indistinguishable from the original anonymity game. Therefore, advantage of adversary in original anonymity game is negligible.

\subsection{Framing Attacks}

- Theorem 3: Our scheme is secure against framing attacks based on the hardness of SIS assumption

- Proof: Let $A$ be an adversary that generates a forgery $\left(m^{*}, \Sigma^{*}\right)$ which opens to the honest user $i^{*}$ who did not sign the message $m^{*}$. We construct an algorithm $B$ that solves an instance of SIS assumption i.e., given a matrix $\bar{A} \in Z_{q}^{4 n \times 4 m}$ as input, algorithm $B$ finds the vector $x$ such that $\bar{A} x=0 \bmod q$ and $\|x\| \leq 2 \beta \sqrt{m}$.

Algorithm $B$

- Setup: Obtain (gpk,gmsk) using $\operatorname{Setup}\left(1^{n}\right)$ with one modification. Instead of uniformly choosing $F \in$ $Z_{q}^{4 n \times 4 m}$, we assign $F=\bar{A}$.

Queries:

- $Q_{Y}:$ returns the public-keygpk.

- $Q_{s}$ : returns gmsk to $A$

- $Q_{b-j o i n}: A$ can corrupt the group manager and introduces new user through $Q_{b \text {-join }}$ protocol. At each query, $B$ runs join protocol on behalf on the honest user $U_{i}$.

- $Q_{\text {sig }}$ : If $A$ requests for the signature on message $m$ of user $I$ and $i \in U^{(b)}$ then, recall $\left(\operatorname{cert}_{i}\right.$, sec $\left._{i}\right)$ and generate signature using $\operatorname{Sign}\left({\left.\text { gpk }, \text { Sec }_{i}, \text { Cert }_{i}, m\right)}\right.$ algorithm.

- Forgery: Let $A$ outputs $\left(m^{*}, \Sigma^{*}, \tau^{*}\right)$ such that Verify $\left(g p k, m^{*}, \Sigma^{*}\right)=1$ with non-negligible $\begin{array}{llll}\text { probability } & \varepsilon \text {. } & \text { Let } & \Sigma^{*}=\end{array}$ $\left(c_{v^{*}}, c_{w^{*}}, \Pi^{*}, V K^{*}, G_{3}^{*}, b_{1}^{*}, \operatorname{sig}^{*}\right)$.Obtain witness $\left(j, d_{j}=\left[d_{1 j} \| d_{2 j}\right], z^{*}, s^{*}\right)$ using witness extraction procedure similar to the steps in misidentification attack (coin=1). We consider three cases where $A$ returns 1 in $\operatorname{Exp}_{f r a}^{\mathrm{A}}(n)$ and show that $B$ solves SIS instance in all these cases.

- $\operatorname{Open}\left(\Sigma^{*}, g p k, g m s k\right)=i^{*} \in U^{(b)}$

- $\forall_{i \in U^{(b)}} \operatorname{Trace}\left(\Sigma^{*}, \operatorname{Reveal}(i)\right)=1$

- $\forall_{i \in U^{(b)}}\left(i, \Sigma^{*}\right) \in$ Sigs and Claim-Verify $\left(\Sigma^{*}, \tau^{*}\right)=1$

- Case1: Open algorithm decrypts and obtain the vector $v_{i *}$. Recall $z_{i *}$ when answering $Q_{b \text {-join }}$ query such that $F z_{i^{*}}=v_{i *}$. We know $v_{i^{*}=} F z^{*}$. In adversary view, $z_{i^{*}}$ is chosen according to $\mathrm{D}_{\Lambda^{\perp}} v_{i^{*}}(F), \sigma$, it has atleast $n$ bits of min-entropy. Therefore, $\mathrm{x}=z^{*}-z_{i *}$ is a solution to SIS instance i.e., $F x=0 \bmod q$ and $x \leq 2 \beta \sqrt{m}$.

- Case2: $\operatorname{Trace}\left(\Sigma^{*}, \operatorname{Reveal}\left(j^{*}\right)\right)=1$ where $j^{*} \in U^{(b)}$ then, $D_{0} \operatorname{bin}\left(v_{i^{*}}\right)+D_{1} S_{i^{*}}=D_{0} \operatorname{bin}\left(v_{j^{*}}\right)+D_{1} S_{j^{*}}$ This is possible only if $v_{i^{*}}=v_{j^{*}}$ and $s_{i^{*}}=s_{j^{*}}$. If $v_{i^{*}}=$ $v_{j^{*}}$ then $F z_{i^{*}}=F z_{j^{*}}$. Therefore, $x=z_{i^{*}}-z_{j^{*}}$ is a solution to SIS instance.

- Case3: If $\forall_{i \in U^{(b)}}\left(i, \Sigma^{*}\right) \in$ Sigs and Claim $\operatorname{Verify}\left(\Sigma^{*}, \tau^{*}\right)=1$ Let $\left(j^{*}, \Sigma^{*}\right) \in$ Sigs recall $z_{j^{*}}$ from $Q_{b^{-} \text {join }}$ protocol such that $v_{j^{*}=} F z_{j^{*}}$. Using improved forking lemma and witness extractor procedure, obtain $z^{*}$ from $\tau^{*}$ such that $v_{j^{*}=} F z^{*}$. Let $x=z_{j *-} z^{*}$ which is a solution to our SIS instance.

\section{Conclusions}

This work presents the first traceable signature scheme based on lattices. Compared to the existing lattice- 
based schemes our scheme has additional features like signature claiming and user opening. As agents can run in parallel, user tracing is scalable. Our scheme is based on the work of [12]. Compared to the scheme in [12], our scheme supports signature claiming, user opening and size of $g p k$ is efficient by $\log N$ factor, where $N$ is the number of members in the group. Our scheme is proved to be secure based on LWE and SIS assumptions in random oracle model. Construction of lattice-based traceable signature without random-oracle is the future work.

\section{References}

[1] Agrawal S., Boneh D., and Boyen X., "Efficient Lattice (H) IBE in the Standard Model," in Proceedings of Annual International Conference on the Theory and Applications of Cryptographic Techniques, Monaco and Nice, pp. 553-572, 2010.

[2] Alwen J. and Peikert C., "Generating Shorter Bases for Hard Random Lattices" Theory of Computing Systems, vol. 48, no. 3, pp. 535-553, 2011.

[3] Boyen X., "Lattice Mixing and Vanishing Trapdoors: A Framework for Fully Secure Short Signatures and More," in Proceedings of in International Workshop on Public Key Cryptography, Paris, pp. 499-517, 2010.

[4] Brickell E., Pointcheval D., Vaudenay S., and Yung M., "Design Validations for Discrete Logarithm Based Signature Schemes," in Proceedings of the International Workshop on Practice and Theory in Public Key Cryptography, Melbourne, pp. 276-292, 2000.

[5] Chaum D. and Heyst E, "Group Signatures," in Proceedings of Workshop on the Theory and Application of Cryptographic Techniques, Brighton, pp. 257-265, 1991.

[6] Fiat A. and Shamir A., "How to Prove Yourself: Practical Solutions to Identification and Signature Problems," in Proceedings of Conference on the Theory and Application of Cryptographic Techniques, Santa Barbara, pp. 186-194, 1986.

[7] Gentry C., Peikert C., and Vaikuntanathan V., "Trapdoors for Hard Lattices and New Cryptographic Constructions," in Proceedings of the fortieth annual ACM Symposium on Theory of Computing, New York, pp. 197-206, 2008.

[8] Gordon S., Katz J., and Vaikuntanathan V., "A Group Signature Scheme from Lattice Assumptions," in Proceedings of International Conference on the Theory and Application of Cryptology and Information Security, Singapore, pp. 395-412, 2010.

[9] Kiayias A., Tsiounis Y., and Yung M., "Traceables Signatures," in Proceedings of
International Conference on the Theory and Applications of Cryptographic Techniques, Interlaken, pp. 571-589, 2004.

[10] Laguillaumie F., Langlois A., Libert B., and Stehlé D., "Lattice Based Group Signatures with Logarithmic Signature Size," in Proceedings of International Conference on the Theory and Application of Cryptology and Information Security, Bengaluru, pp. 41-61, 2013.

[11] Langlois A., Ling S., Nguyen K., and Wang H., "Lattice-based Group Signature Scheme with Verifier-Local Revocation," in Proceedings of International Workshop on Public Key Cryptography, Buenos Aires, pp. 345-361, 2014.

[12] Libert B., Ling S., Mouhartem F., Nguyen K., and Wang H., "Signature Schemes with Efficient Protocols and Dynamic Group Signatures from Lattice Assumptions," in Proceedings of International Conference on the Theory and Application of Cryptology and Information Security, Hanoi, pp. 373-403, 2016.

[13] Libert B., Mouhartem F., and Nguyen K., "A Lattice-Based Group Signature Scheme With Message-Dependent Opening," in Proceedings of International Conference on Applied Cryptography and Network Security, London, pp. 137-155, 2016.

[14] Ling S., Nguyen K., Wang H., and Xu Y., "Lattice-Based Group Signatures: Achieving Full Dynamicity with Ease," in Proceedings of International Conference on Applied Cryptography and Network Security, Kanazawa, pp. 293-312, 2017.

[15] Regev O., "On Lattices, Learning with Errors, Random Linear Codes, and Cryptography," Journal of the ACM, vol. 56, no. 6, pp. 1-37, 2009.

[16] Sakthivel A., "Enhancing Cloud Security based on Group Signature," The International Arab Journal on Information Technology," vol. 14, no. 6, pp. 923-929, 2017.

[17] Shor P., "Polynomial-Time Algorithms for Prime Factorization and Discrete Logarithms on a Quantum Computer," Society for Industrial and Applied Mathematics Journal, vol. 41, no. 2 pp. 1484-1509, 1997. 


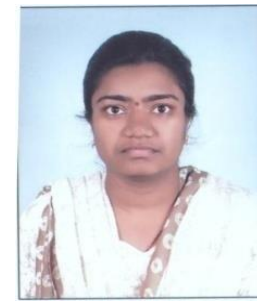

Thakkalapally

Preethi

Thakkalapally Preethi is pursuing her $\mathrm{PhD}$ in Computer Science and Engineering at National Institute of Technology Warangal, India. She received her $\mathrm{M}$ Tech in Computer Science (CS) from University of Hyderabad, India in 2014. Her areas of interest are lattice-based cryptography, digital signatures, provable security and algorithms.

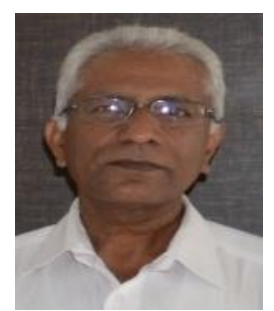

Bharat Amberker received his $\mathrm{PhD}$ in 1996 from Indian Institute of Science (IISc), Bangalore, India from the Department of Computer Science and Automation. $\mathrm{He}$ is presently working as a Professor in Computer Science and Engineering, National Institute of Technology (NIT) Warangal. He is a senior member IEEE, senior member ACM and member of Cryptology Research Society of India. He has guided PhDs in the area of cryptography and security. His research interest includes cryptography, provable security of cryptographic protocols/primitives, algorithms, information security, network security and digital image watermarking. 